\title{
Modifications in anesthesia for geriatric patients
}

\author{
Brandon Chau (Meds 2018), Alexander Levit (MD/PhD 2020) \\ Faculty Reviewer: Dr Daniel Cuillerier, MD, FRCPC (Department of Anesthesia and Perioperative Medicine)
}

\section{ABSTRACT}

The volume of geriatric surgery is expected to increase dramatically by 2020, requiring a more widespread appreciation of the unique risks and challenges of anesthesia in the elderly. Changes in pharmacokinetics along with age-related changes in organ function have important implications for patient monitoring and dosing of anesthetic, analgesic, and sedative medications. Preoperative screening for risk of postoperative morbidity is improved with an assessment of activities of daily living, and regional anesthesia may be considered to reduce the risk of postoperative delirium, although this remains controversial. Specific homeostatic parameters should be closely monitored in the perioperative period. The approach to anesthesia in geriatric patients should not be merely extrapolated from younger patients, and further evidence specific to geriatric anesthesia will improve surgical outcomes.

It is no secret that our population is aging, with an estimated $50 \%$ increase in the over- 65 population by 2020 from 2001 . Older patients also have invasive procedures at nearly quadruple the rate of younger patients, particularly in ophthalmology and cardiology (9- and 2.5-fold, respectively). ${ }^{1,2}$

Across a variety of surgical procedures, a linear increase in the absolute risk of surgical morbidity is observed with age, increasing from $11 \%$ in $20-29$ year old patients to $49 \%$ in octogenarians. Surgical mortality increases exponentially with age, with a dramatic increase from the fifth decade of life, reaching $6 \%$ in octogenarians as compared to $0.4 \%$ in patients in the $20-29$ year old cohort. While the prevalence of risk factors is correlated to age, age remains an independent risk factor for postoperative morbidity and mortality. ${ }^{3}$

As human physiology changes with age, it has been widely shown that pharmaceutical drugs have correspondingly modulated pharmacokinetics. The drugs in the anesthesiologist's arsenal are no exception, and this article will review concerns that the anesthesiologist must take into consideration when treating the geriatric patient. More specifically, this article will discuss considerations for planned surgeries, rather than emergent operations, in order to focus on the importance of preoperative screening and proper follow-up.

\section{PHYSIOLOGICAL CHANGES OF AGING}

\section{Pharmacokinetic changes in drugs}

There are important age-associated body compositional changes with implications for pharmacokinetics. Reduced relative water content and increased fat percentage result in a higher serum drug concentration after bolus injection and longer half-lives for lipid-soluble drugs. Declining cardiovascular function can slow drug delivery to tissue sites, resulting in a slower onset of action.
Decreased albumin content and protein binding results in a higher volume of distribution of acidic drugs. The opposite is seen for alkaline drugs. ${ }^{4}$

As the human body ages, gradual deterioration of all body systems also results in reduced hepatic and renal metabolism and excretion, impaired cardiovascular distribution and disrupted fluid homeostasis. Although the liver and kidneys display minimal structural changes with aging, it has been found that P450-dependent oxidation decreases, meaning that hepatically cleared drugs (eg propofol) have a longer half-life. ${ }^{4}$

\section{Pharmacodynamic changes in drugs}

Decline in cardiovascular health, especially through stiffening of the blood vessels and cardiac decline, is a leading risk factor for peri- and postoperative morbidity. Cardiac decline describes diastolic dysfunction and an increasing dependence on sympathetic drive and the Frank-Starling mechanism. Increased central nervous system dependence of cardiac function often manifests as a marked decline in blood pressure with anesthesia induction. ${ }^{4}$

Inhaled anesthetic agents such as sevoflurane and desflurane remain agents of choice for North American anesthesiologists, despite the still-unclear mechanism of action. It is therefore important to recognize that inhaled drugs have a decreased minimum alveolar concentration (MAC) in geriatric patients due to a greater sensitivity; whereas the MAC of isoflurane in the pediatric patient is $1 \%$, it is $0.25 \%$ in the 95 -year-old patient. Geriatric patients also exhibit a higher sensitivity to opioids and benzodiazepines, so it is important for the anesthesiologist to carefully titrate administration of narcotics to minimize toxicity while maintaining effective pain management. ${ }^{4,5}$

\section{PRACTICAL CONSIDERATIONS IN THE OR}

Since geriatric patients are often receiving polypharmaceutical treatment, it is important to conduct physiologic optimization and to minimize premedications (eg heparin, diuretics). Preoperative risk factors (eg hypertension, dyspnea) have been identified to be more important than intraoperative events (eg transient hypoxemia, apnea) in predicting postoperative morbidity.

While preoperative screening is common practice in most centres, it is important to be more thorough with elderly patients and to complete a comprehensive history and physical exam. Many abnormal lab values, including anemia, creatinine, and hyperglycemia, are less effective than history and physical examination at predicting postoperative morbidity. ${ }^{6,7}$ To that end, a geriatric assessment is recommended for elderly surgical patients; 6 questions regarding activities of daily living and instrumental activities of daily living have been shown to identify $98.5 \%$ of elders with postoperative morbidity (Table 1$){ }^{8}$ 
Table 1: Sample 6-Item Pre-Op Questionnaire ${ }^{6}$

\begin{tabular}{l}
\hline Ascending/descending stairs \\
\hline Urine continence \\
\hline Walking in a corridor \\
\hline Bathing \\
\hline Shopping \\
\hline Food preparation \\
\hline
\end{tabular}

\section{Perioperative considerations and patient management}

Control of body temperature is essential for successful surgery as anesthesia can lead to hypothermia. This effect is further pronounced in the geriatric patient because baseline hypothalamic function often declines, leading to impaired thermoregulation, especially with general anesthesia. Since even mild hypothermia can lead to infection or myocardial infarction, the anesthesiologist must be particularly vigilant with maintenance of body heat in the elderly. ${ }^{9}$

Because age-related deterioration of renal and cardiac function negatively impacts fluid homeostasis, it is prudent to carefully monitor blood pressure when administrating IV fluids and to be watchful for renal overload. It is particularly important to meticulously pay attention to water balance, drug dosing, and maintenance of normal intravascular volume to keep patients healthy. ${ }^{10}$

Changes in pulmonary physiology also result in increased physiologic dead space due to compliant airways, so minute ventilation should be increased, and positive end-expiratory pressure can also be considered. Nasal cannulas are also indicated for virtually all elderly patients due to declining respiratory function and reserve. ${ }^{9}$

As suggested above, the recommended doses for drugs are nearly globally decreased in the elderly. A more comprehensive table is provided (Table 2 ). ${ }^{5}$

Table 2: Recommendations for Dosage Adjustment Consequent to Altered Pharmacokinetics and Pharmacodynamics in the Elderly ${ }^{5}$

\begin{tabular}{|c|c|}
\hline Class of drug & Dosage adjustment \\
\hline Barbiturates & Modest reduction in bolus dose and infusion rates \\
\hline Etomidate & Reduction of up to $50 \%$ in bolus dose \\
\hline Propofol & Reduction of $30-50 \%$ in bolus dose and infusion rates \\
\hline Benzodiazepines & Reduction of up to $75 \%$ in bolus dose and infusion rates \\
\hline Opioids & Reduction of up to $50 \%$ in bolus dose and infusion rates \\
\hline $\begin{array}{l}\text { Non-depolarizing } \\
\text { neuromuscular } \\
\text { blocking agents }\end{array}$ & $\begin{array}{l}\text { No reduction in bolus dose, but generally reduced infu- } \\
\text { sion rates depending on the drug }\end{array}$ \\
\hline Volatile agents & $\begin{array}{l}\text { Reduction in inspired concentrations of } 6 \% \text { per decade } \\
\text { of age }\end{array}$ \\
\hline Local anesthetics & Small to moderate reduction in segmental dose \\
\hline
\end{tabular}

\section{Postoperative monitoring}

It is important to be aware of postoperative morbidities such as cardiovascular damage and stroke. Intraoperative complications, which happen in $20 \%$ of geriatric patients, lead to a 6 -fold increase in morbidity. Hospitalization has also been identified as a risk factor for postoperative morbidity, so when possible, outpatient surgeries are especially recommended for geriatric patients as this generally reduces nosocomial risk in all patients. Finally, since delirium and dementia can also occur in the geriatric population after anesthesia, it is important to monitor for changes in mental status during follow-up. ${ }^{7,11}$

\section{CONCLUSION}

With the geriatric population projected to increase, compounded with an increasing rate of surgery and of morbidity/mortality risk in patients over 65 , it is important to understand the concerns about anesthesia in elderly patients and to appreciate where extra vigilance is required. Due to a global decline in physiological function and reserve, drug pharmacokinetics are often severely affected. Declining fluid and temperature homeostasis also imposes more responsibilities on the anesthesiologist. Finally, certain classes of drugs, such as opioids and inhaled anesthetics, demonstrate a markedly increased potency in the elderly patient, necessitating careful titration to avoid toxicity. The field of geriatric anesthesia is increasing in importance because extrapolation of knowledge from younger patients may not accurately apply to elderly patients. Best practice guidelines may need to change as more geriatric-specific evidence emerges in the coming years.

\section{REFERENCES}

1. Etzioni DA, Liu JH, Maggard MA, Ko CY. The aging population and its impact on the surgery workforce. Ann Surg. 2003 Aug;238(2):170-7.

2. Rooke, GA. Cardiovascular aging and anesthetic implications. J Cardiothorac and Vasc Anesth. 2003 Aug;17(4):512-23.

3. Turrentine FE, Hongkun W, Simpson VB, Jones RS. Surgical risk factors, morbidity, and mortality in elderly patients. J Am Coll Surg. 2006 Dec;203(6):86577.

4. Sadean, Mihai R., and Peter SA Glass. Pharmacokinetics in the elderly. Best Pract Res Clin Anaesthesiol. 2003 Jun;17(2):191-205.

5. Nickalls RWD, Mapleson WW. Age-related iso-MAC charts for isoflurane, sevoflurane and desflurane in man. Br J Anaesth. 2003 Aug;91(2):170-4.

6. Dzankic S, Pastor D, Gonzalez C, Leung JM. The prevalence and predictive value of abnormal preoperative laboratory tests in elderly surgical patients. Anesth Analg 2001 Aug;93(2):301-8.

7. Liu LL, Leung JM. Predicting adverse postoperative outcomes in patients aged 80 years or older. J Am Geriatr Soc. 2000 Apr;48(4):405-12.

8. Roehrig B, Hoeffken K, Pientka L, Wedding U. How many and which items of activities of daily living (ADL) and instrumental activities of daily living (IADL) are necessary for screening. Crit Rev Oncol Hematol. 2007 May;62(2):164-71.

9. Hart SR, Bordes B, Hart J, Corsino D, Harmon D. Unintended perioperative hypothermia. Ochsner J. 2011 Fall;11(3):259-70.

10. Beck LH. Perioperative renal, fluid, and electrolyte management. Clin Geriatr Med. 1990 Aug;6(3):557-69.

11. Kanonidou Z, Karystianou G. Anesthesia for the elderly. Hippokratia. 2007 Oct-Dec;11(4):175-7. 